\title{
Daytime Sleepiness: Associations with Alcohol Use and Sleep Duration in Americans
}

\author{
Subhajit Chakravorty, ${ }^{1,2}$ Nicholas Jackson, ${ }^{3}$ Ninad Chaudhary, ${ }^{2,4}$ Philip J. Kozak, ${ }^{5}$ \\ Michael L. Perlis, ${ }^{2}$ Holly R. Shue, ${ }^{6}$ and Michael A. Grandner ${ }^{2}$ \\ ${ }^{1}$ MIRECC VISN-4, Philadelphia Veterans Affairs Medical Center, University \& Woodland Avenues, Philadelphia, PA 19104, USA \\ ${ }^{2}$ Perelman School of Medicine, University of Pennsylvania, Philadelphia, PA 19104, USA \\ ${ }^{3}$ University of Southern California, Los Angeles, CA 90033, USA \\ ${ }^{4}$ West Chester University of Pennsylvania, West Chester, PA 19383, USA \\ ${ }^{5}$ School of Veterinary Medicine, University of Pennsylvania, Philadelphia, PA 19104, USA \\ ${ }^{6}$ Children's Hospital of Philadelphia, Philadelphia, PA 19104, USA
}

Correspondence should be addressed to Subhajit Chakravorty; subhajit.chakravorty@uphs.upenn.edu

Received 30 June 2013; Revised 29 October 2013; Accepted 26 November 2013; Published 29 January 2014

Academic Editor: Michel M. Billiard

Copyright (C) 2014 Subhajit Chakravorty et al. This is an open access article distributed under the Creative Commons Attribution License, which permits unrestricted use, distribution, and reproduction in any medium, provided the original work is properly cited.

\begin{abstract}
The aim of the current analysis was to investigate the relationship of daytime sleepiness with alcohol consumption and sleep duration using a population sample of adult Americans. Data was analyzed from adult respondents of the National Health and Nutritional Examination Survey (NHANES) 2007-2008 ( $N=2919)$ using self-reported variables for sleepiness, sleep duration, and alcohol consumption (quantity and frequency of alcohol use). A heavy drinking episode was defined as the consumption of $\geq 5$ standard alcoholic beverages in a day. Logistic regression models adjusted for sociodemographic variables and insomnia covariates were used to evaluate the relationship between daytime sleepiness and an interaction of alcohol consumption variables with sleep duration. The results showed that daytime sleepiness was reported by $15.07 \%$ of the subjects. In univariate analyses adjusted for covariates, an increased probability of daytime sleepiness was predicted by decreased log drinks per day [OR $=0.74(95 \%$ CI, 0.58-0.95)], a decreased log drinking frequency [0.90 (95\% CI, 0.83-0.98)], and lower sleep duration [OR $=0.75$ (95\% CI, 0.67-0.84)]. An interaction between decreased sleep duration and an increased log heavy drinking frequency predicted increased daytime sleepiness $(P=0.004)$. Thus, the effect of sleep duration should be considered when evaluating the relationship between daytime sleepiness and heavy drinking.
\end{abstract}

\section{Introduction}

Daytime sleepiness is highly prevalent. 19.5\% of Americans suffer from moderate sleepiness and $11 \%$ suffer from severe sleepiness [1]. These prevalence rates are of concern given that daytime sleepiness affects nearly every aspect of human functioning and is a substantial risk factor for accidents and injuries [2]. The combination of daytime sleepiness and atrisk vocations vulnerable to such negative effects magnifies the risk for adverse outcomes of accidents, for example, sleepiness in truck drivers, airline pilots, medical personnel, mass transit operators, and so forth [3]. The perils associated with these conditions underscore the need to identify factors that may serve to aggravate sleepiness, or may serve as vulnerability for sleepiness. One such condition may be the use of or the abuse of alcohol.

To date, some studies have been undertaken to evaluate the relationship between daytime sleepiness and alcohol consumption. The outcomes from these studies were inconsistent. Acute drinking episodes were found to be associated with complaints of sleepiness (as a component of hangover symptoms) [4] and an acute impairment in objective measures of flying the following day (after an acute drinking episode) [5]. In contrast, increased alcohol consumption 
( $>7$ drinks per week) has been shown to be associated with a decreased likelihood of excessive daytime sleepiness in the elderly [6]. These studies differed in several ways, including the following: the duration of alcohol use (i.e., acute versus chronic use), the age of the cohort studied, and the sleep duration immediately prior to the study (or the typical total sleep duration).

The last of these issues is the role of sleep duration, which is particularly important as an increased consumption of alcohol has been independently associated with short sleep duration in some prior epidemiological studies [7-9]. Sleep duration has been investigated as a moderator in this relationship between alcohol consumption and sleepiness in a few laboratory-based studies. Some of these studies involved paradigms with partial sleep restriction and some with sleep extension (longer than typical ad lib sleep). In the partial sleep deprivation studies, Rupp and colleagues found that nocturnal alcohol consumption prior to bedtime and in conjunction with a partial sleep restriction increased the sleepiness at night (as compared to those who did not consume alcohol) [10]. Roehrs and colleagues found that despite an increase in the sleep latency on the Multiple Sleep Latency Test (MSLT) with partial sleep deprivation and moderate nocturnal alcohol consumption, no interaction between alcohol consumption and sleep deprivation was seen [11]. Similarly, a study by Horne and colleagues failed to show a difference in daytime sleepiness in the sleep-deprived conditions, with or without afternoon alcohol consumption, by using a different paradigm [12]. In the sleep extension studies, Lumley and colleagues found no differences in objective sleepiness on MSLT in subjects with an 11-hour total time in bed and morning alcohol consumption (as compared to normal sleep or partial sleep deprivation conditions) [13]. In another study, Roehrs and colleagues found that sleep extension with a 10-hour time in bed and morning alcohol consumption showed a decreased objectively measured sleepiness with a MSLT test [14].

As can be seen from the above summary, the data to date are mixed. If trends are evident it appears that heavy alcohol consumption is associated with shorter sleep durations, and objective daytime sleepiness may be associated with acute alcohol use/alcohol abuse in association with sleep deprivation. These findings must be considered tentative, as there are only a few studies with mixed results, and the alcohol measures are not well operationalized. With respect to the alcohol measures, the variables used rarely allow for a comprehensive assessment that takes into account dose (amount of alcohol per occasion), use frequency (number of occasions using alcohol per day or week), and use in the hazardous range (the presence of heavy drinking, and the frequency of heavy drinking as defined as the use of $\geq 5$ drinks per session) $[15,16]$.

Accordingly, using a nationally representative sample, we explored the interactions between self-reported alcohol consumption and sleep duration variables and their association to daytime sleepiness while controlling for covariates and symptoms related to several intrinsic sleep disorders.

\section{Methods}

2.1. Design and Setting. This investigation utilized the 20072008 National Health and Nutrition Examination Survey (NHANES). This annual survey, conducted by the Centers for Disease Control and Prevention, assesses the demographic, health, and nutritional characteristics in the US population through in-person interviews, physical examinations, and laboratory tests. The unweighted response rate for the overall sample was $78.4 \%$. In order to compensate for underrepresentation, African Americans, Hispanics, and adults over 60 were over-sampled [17].

2.2. Sample Size. Out of the initial participants $(N=10,149)$, we excluded children and adolescents $<18$ years of age $(N=$ $3921)$, those with a lifetime history of drug use $(N=1896)$, those without response to outcome variables $(N=21)$, and those missing data on predictor variables $(N=1079)$, and covariates $(N=313)$. The final sample consisted of 2919 subjects. Drug use history was assessed for any lifetime use of marijuana, cocaine, methamphetamine, or illicit opiates individually; the response was coded dichotomously as "yes"/ "no."

\subsection{Measures}

2.3.1. Sleep. (a) Daytime sleepiness (DS) was assessed with the question, "In the past month, how often did you feel excessively or overly sleepy during the day?" (b) Sleep Duration $(S D)$ was investigated with the question, "How much sleep do you usually get at night on weekdays or workdays?" This question was similar to that used in prior studies $[18,19]$. (c) Insomnia symptoms: (i) difficulty falling asleep $(D F A)$ was assessed using the question "In the past month, how often did you have trouble falling asleep?" (ii) difficulty maintaining sleep $(A W A K)$ was assessed with the question "In the past month, how often did you wake up during the night and had trouble getting back to sleep?" (iii) Nonrestorative sleep $(N R S)$ was evaluated with the question,"In the past month, how often did you feel unrested during the day, no matter how many hours of sleep you have had?" The responses to the DS, SL, AWAK, and SQ variables were presented with the following severity options: "0" (never), "1" (rarely: 1 time/ month), "2" (sometimes: 2-4 times/month), "3" (often: 5-15 times/month), and "4" (almost always: 16-30 times/month). The response to the $\mathrm{SD}$ variable was recorded as a number, rounded to the nearest decimal point and was assessed as a continuous variable.

2.3.2. Alcohol-Related Variables. The quantity of alcohol in a drink was evaluated in terms of a standard alcoholic drink [20]. The alcohol consumption variables used in this investigation were in line with those used in prior studies, and included the following [16, 21]: (a) drinks/day: this variable was assessed with "In the past 12 months, on those days that you drank alcoholic beverages, on an average, how many drinks did you have?" (b) drinking frequency was investigated using the question "In the past 12 months, how often did you drink any type of alcoholic beverage?" (c) heavy drinking status was assessed using the question 
"In the past 12 months, on how many days did you have 5 or more drinks of any alcoholic beverage?" (d) heavy drinking frequency was evaluated using the question, "In the past 12 months, on how many days did you have 5 or more drinks of any alcoholic beverage?" Drinks/day, drinking frequency and binge-drinking frequency were recorded continuously as number of days. Heavy drinking status was recorded dichotomously, as "present" or "absent."

2.3.3. Covariates. The variables included in these analyses included, age, gender, race/ethnicity (White, Black/African American, Hispanic, and other), marital status, education, income, body mass index (BMI; objectively measured), depression (over past two weeks), anxiety (days anxious in past month), access to health insurance, physical health, mental health, exercise, and smoking (smoking days in past month). All the above-mentioned questions were assessed as part of the NHANES interview with the responses being selfreported by the subjects.

2.4. Statistical Analysis. Two-year full sample weights were used to adjust for unequal probability of being selected among noncoverage or nonresponse population, as recommended [17]. Daytime sleepiness was assessed with the question, "In the past month, how often did you feel excessively or overly sleepy during the day?" Daytime sleepiness was assessed as a dichotomous variable ("presence" or "absence" of daytime sleepiness) based on the distribution of the response. The sleep duration was assessed as a continuous variable. Alcohol consumption was assessed using four variables, including, drinks/day, drinking frequency, heavy drinking status, and heavy drinking frequency. Heavy drinking status was assessed as a dichotomous variable ("presence" or "absence" of heavy drinking). The remaining 3 alcohol variables were assessed as continuous variables, drinks/day, drinking frequency, and heavy drinking frequency. $\log _{n}$ transformation was conducted for the variables including, drinks/day, drinking frequency, and heavy drinking frequency because of the skewness in the data, prior to the bivariate and multivariable analyses. Some of the covariates were dichotomized because of the skewed distribution and included insomnia variables (reporting a complaint $\geq 5$ times a month $/<5$ times a month), depression, and anxiety symptoms (symptoms $<15$ days/ $\geq 15$ days over last month). The relationships between daytime sleepiness (dependent variable) and alcohol consumption variables were assessed using multinomial logistic regression analyses. This relationship was assessed using three different models to adjust for covariates. Model 1 assessed the crude relationship between daytime sleepiness and the alcohol consumption variables or sleep duration. Model 2 assessed the relationship in model 1, adjusted for the covariates mentioned above. Model 3 assessed for this relationship in Model 2, further adjusted for insomnia symptoms. Interactive models evaluated for the presence of 2-way interactions, of whether the effects of one alcohol consumption variable depended on levels of a second category, that is, the sleep duration in predicting daytime sleepiness. Analyses were conducted using Stata version 12
(StataCorp LP, Stata Statistical Software: Release 12. College Station, TX).

\section{Results}

3.1. Subjects. The average subject in this study which was middle-aged female, college graduate, who identified herself as of Caucasian race, non-Hispanic in ethnicity, married, was overweight and had health insurance, Table 1.

3.2. Sleep-Related Characteristics. Daytime sleepiness was reported by $15.07 \%$ of the subjects. The mean (SD) sleep duration was $6.91(\mathrm{SD}=1.36$ ) hours. Amongst them, 56.72\% had sleep duration within the normal range (7-8 hours a night), $36.12 \%$ had short sleep duration ( $\leq 6$ hours a night), and $7.16 \%$ had long sleep duration ( $\geq 8$ hours a night), with nonrestorative sleep being the commonest insomnia symptom $(22.32 \%)$, Table 1.

3.3. Alcohol Consumption. The average subject reported an alcohol consumption in the moderate range with a mean (SD) alcohol consumption of $1.25(\mathrm{SD}=2.28)$ drinks per day within the last 12 months. Amongst those drinking alcohol in the past 12 months, $9.77 \%$ of the respondents reported heavy drinking ( $\geq 5$ drinks a day), and with a heavy drinking frequency of $4.23(\mathrm{SD}=27.18)$ days of over the last 12 months, Table 1.

\subsection{The Relationship of Daytime Sleepiness with Alcohol Consumption and Sleep Duration}

3.4.1. Alcohol Consumption. Subjects with daytime sleepiness reported lower alcohol consumption as compared to those without daytime sleepiness $(0.99 \pm 1.65$ drinks and $1.29 \pm 2.38$ drinks resp., $P=0.002$ ). In analyses adjusted for sociodemographic variables and insomnia covariates, a decreased risk of daytime sleepiness was predicted by log drinks per day, that is, each percent increase in the number of alcoholic drinks per day $[\mathrm{OR}=0.74(95 \% \mathrm{CI}, 0.58-0.95), P=0.019]$. A similar relationship of a decreased risk of daytime sleepiness was seen with increased log drinking frequency, that is, each percent increase in the frequency of drinking and with nonsignificant trends for log binge-drinking frequency predicting a lower risk of daytime sleepiness, Table 2 .

3.4.2. Sleep Duration. Those with daytime sleepiness reported a lower sleep duration as compared to those without daytime sleepiness $(6.38 \pm 1.63$ hours and $7.00 \pm 1.28$ hours, resp., $P<0.0001)$. In analyses adjusted for sociodemographic variables and insomnia covariates, a decreased probability of daytime sleepiness was predicted by higher sleep duration [OR $=0.75$ (95\% CI, 0.67-0.84), $P<0.001]$, Table 2 .

3.5. Interactions between Alcohol Consumption and Sleep Duration on Daytime Sleepiness. In models adjusted for covariates, an interaction between a decreased sleep duration and an increased log frequency of binge-drinking predicted increased daytime sleepiness $(P=0.004)$, such that with each percent increase in the binge-drinking frequency and 
TABLE 1: Baseline demographics.

\begin{tabular}{|c|c|c|c|}
\hline Variable & Categories & Mean/\% & S.D. \\
\hline Age (years) & & 53.1 & 17.4 \\
\hline Gender & Female & $57.84 \%$ & \\
\hline \multirow{3}{*}{ Race } & White & $68.05 \%$ & \\
\hline & Black & $10.20 \%$ & \\
\hline & Other & $21.76 \%$ & \\
\hline Ethnicity & Hispanic & $14.71 \%$ & \\
\hline $\mathrm{BMI}\left(\mathrm{Kg} / \mathrm{m}^{2}\right)$ & & 29.1 & 6.6 \\
\hline Mental health & Days with poor mental health (past month) & 3.21 & 7.30 \\
\hline Anxiety & $\geq 15$ days (past month) & $12.61 \%$ & \\
\hline Depression & $\geq 15$ days (past month) & $6.01 \%$ & \\
\hline Exercise (in minutes) & Moderate/vigorous exercise & 124 & 187 \\
\hline \multirow{4}{*}{ Education } & College graduate & $26.51 \%$ & \\
\hline & Less than high school & $21.30 \%$ & \\
\hline & High school graduate & $25.88 \%$ & \\
\hline & Some college education & $26.31 \%$ & \\
\hline \multirow{8}{*}{ Income (per year) } & $>75,000$ & $29.79 \%$ & \\
\hline & $<20,000$ & $17.24 \%$ & \\
\hline & $20,000-25,000$ & $7.65 \%$ & \\
\hline & $25,000-35,000$ & $12.45 \%$ & \\
\hline & $35,000-45,000$ & $10.40 \%$ & \\
\hline & $45,000-55,000$ & $8.78 \%$ & \\
\hline & $55,000-65,000$ & $7.03 \%$ & \\
\hline & $65,000-75,000$ & $6.66 \%$ & \\
\hline \multirow{5}{*}{ General heath } & Excellent & $16.77 \%$ & \\
\hline & Very good & $29.57 \%$ & \\
\hline & Good & $34.31 \%$ & \\
\hline & Poor & $15.80 \%$ & \\
\hline & Very poor & $3.55 \%$ & \\
\hline \multirow{5}{*}{ Marital status } & Married & $63.04 \%$ & \\
\hline & Widowed & $9.43 \%$ & \\
\hline & Divorced/separated & $11.11 \%$ & \\
\hline & Never married & $12.29 \%$ & \\
\hline & Living with partner & $4.13 \%$ & \\
\hline Insurance status & Insured & $85.35 \%$ & \\
\hline Caffeine use & Present & $91.06 \%$ & \\
\hline Smoker & Yes & $11.03 \%$ & \\
\hline Daytime sleepiness & Present & $15.07 \%$ & \\
\hline Sleep duration & Sleep duration (hrs) & 6.91 & 1.36 \\
\hline \multirow{3}{*}{ Insomnia symptoms } & Difficulty falling asleep (DFA, $\geq 5$ nights/month) & $16.05 \%$ & \\
\hline & Difficulty maintaining sleep (AWAK, $\geq 5$ nights/month) & $18.15 \%$ & \\
\hline & Nonrestorative sleep (NRS, $\geq 5$ nights/month) & $22.32 \%$ & \\
\hline Alcohol quantity & Drinks/day (past 12 months) & 1.25 & 2.28 \\
\hline Drinking frequency & Drinking days (past 12 months) & 40.9 & 85.8 \\
\hline Heavy drinking status & Present (past 12 months) & $9.77 \%$ & \\
\hline Heavy drinking frequency & Heavy drinking days (past 12 months) & 4.23 & 27.18 \\
\hline
\end{tabular}

S.D.: standard deviation. 
TABLE 2: Associations of daytime sleepiness with alcohol variables and sleep duration.

\begin{tabular}{lccccccc}
\hline \multirow{2}{*}{ Predictor } & \multirow{2}{*}{ Subgroup } & Model 1 & Model 2 & \multicolumn{2}{c}{ Model 3 } \\
& & OR (95\% CI) & $P$ & OR (95\% CI $)$ & $P$ & OR (95\% CI) & $P$ \\
\hline \multirow{3}{*}{ Alcohol } & Log drinks/day & $0.72(0.58-0.90)$ & 0.0046 & $0.78(0.60-1.01)$ & 0.0557 & $0.74(0.58-0.95)$ & 0.0197 \\
& Log drinking frequency & $0.87(0.81-0.93)$ & 0.0001 & $0.91(0.84-0.99)$ & 0.0297 & $0.90(0.83-0.98)$ & 0.0129 \\
& Heavy drinking status & $0.68(0.43-1.07)$ & 0.0971 & $0.76(0.48-1.21)$ & 0.2471 & $0.71(0.45-1.11)$ & 0.1311 \\
Sleep duration & Log heavy drinking frequency & $0.87(0.74-1.02)$ & 0.0805 & $0.89(0.75-1.06)$ & 0.1891 & $0.88(0.75-1.04)$ & 0.1366 \\
& Hours & $0.71(0.64-0.80)$ & $<0.0001$ & $0.75(0.67-0.84)$ & $<0.0001$ & $0.75(0.67-0.84)$ & $<0.0001$ \\
\hline
\end{tabular}

OR: odds ratio, CI: confidence interval, $P$ : $P$ value, and mo: months.

Model 1: unadjusted model.

Model 2: adjusted for age, BMI, gender, race, marital status, education, income, depression, insurance, health status, anxiety, mental health, and exercise. Model 3: model $2+$ insomnia status.

TABLE 3: Daytime sleepiness and its association with an interaction of alcohol consumption variables on sleep duration.

\begin{tabular}{lccc}
\hline Moderating variable & Model 1 $(P)$ & Model 2 $(P)$ & Model 3 $(P)$ \\
\hline Log drinks/day & 0.131 & 0.627 & 0.626 \\
Log drinking frequency & 0.323 & 0.628 & 0.602 \\
Heavy drinking status & 0.059 & 0.286 & 0.289 \\
Log heavy drinking frequency & $\mathbf{0 . 0 0 5}$ & $\mathbf{0 . 0 0 3}$ & $\mathbf{0 . 0 0 4}$ \\
\hline
\end{tabular}

$P: P$ value.

Model 1: unadjusted model.

Model 2: adjusted for age, BMI, gender, race, marital status, education, income, depression, insurance, health status, anxiety, mental health, and exercise.

Model 3: model $2+$ insomnia status.

a decrease in the sleep duration in hours, there was an increased probability of reporting daytime sleepiness; see Table 3, and Figure 1. No significant interactions between other alcohol consumption variables and sleep duration predicted daytime sleepiness.

\section{Discussion}

The association between subjective sleepiness as it relates to alcohol consumption and sleep duration from a population perspective is currently unknown. In this study, we explored this relationship using data from the 2007-2008 NHANES survey using self-reported measures. In univariate analyses, the presence of daytime sleepiness was inversely associated with the drinks per day, drinking frequency, and sleep duration. In the final model adjusted for covariates, an interaction between heavy drinking frequency and sleep duration predicted daytime sleepiness, such that an increased probability of daytime sleepiness was reported with each percent increase in the frequency of heavy drinking and a decrease in the sleep duration (in hours).

Short sleep duration has been linked with heavy alcohol consumption on one hand [7-9] and with daytime drowsiness on the other hand $[22,23]$. Heavy alcohol consumption has been linked with next day symptoms of tiredness [24], and with an impaired performance [5]. It is therefore possible that sleepiness is reliably produced with a higher intensity and periodicity of alcohol consumption along with insufficient habitual sleep duration. The sleep duration may be decreased by the heavy alcohol consumption itself, or from insufficiency based on the need or opportunity of functioning in a 24hour society, and/or the presence of intrinsic sleep disorders like insomnia or obstructive sleep apnea syndrome. In light

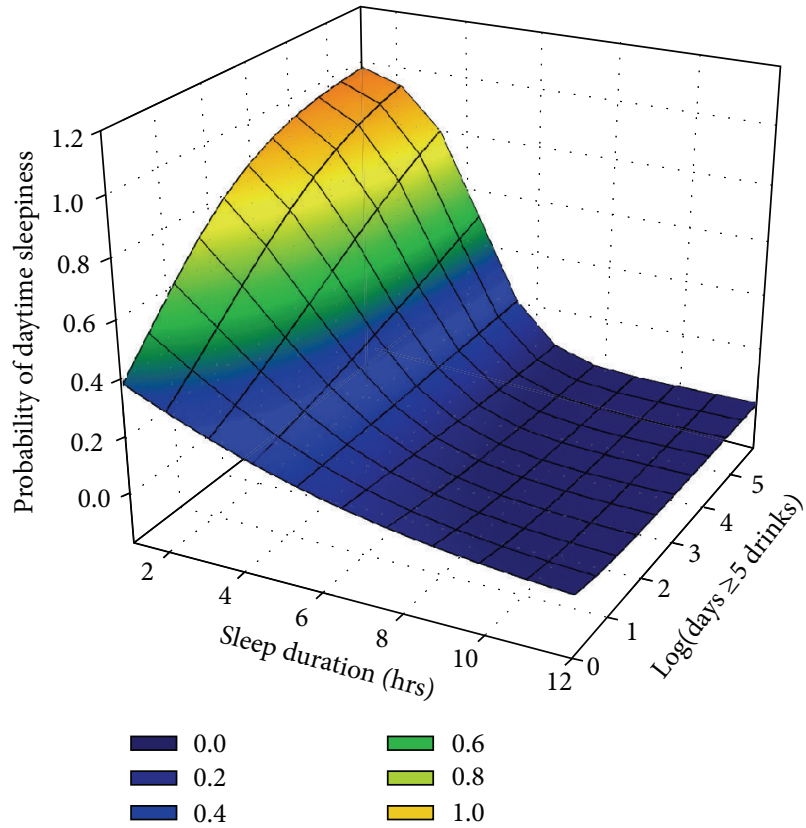

FIGURE 1: Surface model of the interaction between heavy drinking frequency and sleep duration on daytime sleepiness.

of the above it is easier to comprehend our findings of an interaction between heavy drinking and a decreased sleep duration predicting sleepiness.

Evaluating this relationship from another perspective, it is possible that after the alcohol is metabolized in the latter half of the night, the sleep is shallow and fragmented sleep as shown previously $[24,25]$. This shallow and/or fragmented 
sleep may lead the subject to a state of subacute sleep deprivation with continued heavy drinking over time, leading to complaints of daytime sleepiness [4]. Our results differ from those of Pack and colleagues [6] as their study did not account for the sleep duration or for any heavy drinking and showed results similar to our bivariate analysis. In addition, our study adjusted for the effect of gender as a covariate, as well as body mass index and insomnia symptoms in the analyses.

Some of the limitations associated with this study include the following: the cross-sectional nature of the study precludes determination of the cause and effect between the variables; the dichotomous nature of sleepiness complaint prevents us from differentiating relationships associated with varying intensities of the daytime sleepiness; the lack of additional data on the drinking pattern across genders (in a calendar format) over the past year (in days, months, or years) or the pattern of drinking on weekdays versus weekends; and the lack of data on the circadian pattern of sleep and sleepiness as well as caffeine and alcohol consumption. Despite its weaknesses, this is one of the first studies at the population level that shows the presence of a complex relationship between alcohol consumption and sleep duration, on daytime sleepiness.

In conclusion, an inverse relationship of the probability of daytime sleepiness with the intensity and the frequency of alcohol consumption was seen in adult respondents from a nationally representative US sample. Once the duration of sleep was factored in, an interaction between the frequency of heavy drinking and sleep duration predicted an increased probability of daytime sleepiness. These results extend the findings from prior laboratory-based studies to a population sample. Future studies will need to further clarify this complex relationship further using more detailed information on alcohol use, sleepiness in the context of circadian phase and the ascending versus the descending limbs of alcohol concentrations as seen in a prior laboratory study [26], and the association with hangover symptoms. In addition, studies are also warranted to tease apart the role of gender differences in this relationship considering the gender related differences in sleepiness and alcohol consumption.

\section{Conflict of Interests}

None of the authors (Subhajit Chakravorty, Nicholas Jackson, Michael A. Grandner, Ninad Chaudhary, Philip J. Kozak, Holly R. Shue, and Michael L. Perlis) have reported any actual or any potential conflict of interests with the subject matter of the paper.

\section{Authors' Contribution}

Subhajit Chakravorty conceptualized, analyzed, and drafted the paper. Nicholas Jackson worked in data analyses and collaborated with the drafting of this paper. Ninad Chaudhary collaborated with the drafting of this paper. Philip J. Kozak collaborated with the drafting of this paper. Michael L. Perlis collaborated in conceptualization and drafting this paper. Holly R. Shue collaborated with the drafting of this paper.
Michael A. Grandner collaborated in conceptualizing the study, analyzing the data, and drafting of this paper.

\section{Acknowledgments}

The content of this publication does not represent the views of the Department of Veterans Affairs, the United States Government, or any of the collaborating institutions.

\section{References}

[1] M. M. Ohayon, "Determining the level of sleepiness in the American population and its correlates," Journal of Psychiatric Research, vol. 46, no. 4, pp. 422-427, 2012.

[2] T. B. Young, "Epidemiology of daytime sleepiness: definitions, symptomatology, and prevalence," Journal of Clinical Psychiatry, vol. 65, no. 16, pp. 12-16, 2004.

[3] J. S. Durmer and D. F. Dinges, "Neurocognitive consequences of sleep deprivation," Seminars in Neurology, vol. 25, no. 1, pp. 117-129, 2005.

[4] R. Penning, A. McKinney, and J. C. Verster, "Alcohol hangover symptoms and their contribution to the overall hangover severity," Alcohol and Alcoholism, vol. 47, no. 3, pp. 248-252, 2012.

[5] J. A. Yesavage and V. O. Leirer, "Hangover effects on aircraft pilots 14 hours after alcohol ingestion: a preliminary report," The American Journal of Psychiatry, vol. 143, no. 12, pp. 1546-1550, 1986.

[6] A. I. Pack, D. F. Dinges, P. R. Gehrman, B. Staley, F. M. Pack, and G. Maislin, "Risk factors for excessive sleepiness in older adults," Annals of Neurology, vol. 59, no. 6, pp. 893-904, 2006.

[7] M. A. Schuckit and L. I. Bernstein, "Sleep time and drinking history: a hypothesis," The American Journal of Psychiatry, vol. 138, no. 4, pp. 528-530, 1981.

[8] C. D. Palmer, G. A. Harrison, and R. W. Hiorns, "Association between smoking and drinking and sleep duration," Annals of Human Biology, vol. 7, no. 2, pp. 103-107, 1980.

[9] J. P. Chaput, J. McNeil, J. P. Despres, C. Bouchard, and A. Tremblay, "Short sleep duration is associated with greater alcohol consumption in adults," Appetite, vol. 59, no. 3, pp. 650655, 2012.

[10] T. L. Rupp, C. Acebo, E. van Reen, and M. A. Carskadon, "Effects of a moderate evening alcohol dose. I: sleepiness," Alcoholism, vol. 31, no. 8, pp. 1358-1364, 2007.

[11] T. Roehrs, J. Yoon, and T. Roth, "Nocturnal and next-day effects of ethanol and basal level of sleepiness," Human Psychopharmacology, vol. 6, no. 4, pp. 307-311, 1991.

[12] J. A. Horne, L. A. Reyner, and P. R. Barrett, "Driving impairment due to sleepiness is exacerbated by low alcohol intake," Occupational and Environmental Medicine, vol. 60, no. 9, pp. 689-692, 2003.

[13] M. Lumley, T. Roehrs, D. Asker, F. Zorick, and T. Roth, "Ethanol and caffeine effects on daytime sleepiness/alertness," Sleep, vol. 10, no. 4, pp. 306-312, 1987.

[14] T. Roehrs, A. Zwyghuizen-Doorenbos, V. Timms, F. Zorick, and T. Roth, "Sleep extension, enhanced alertness and the sedating effects of ethanol," Pharmacology Biochemistry and Behavior, vol. 34, no. 2, pp. 321-324, 1989.

[15] D. A. Dawson, "Defining risk drinking," Alcohol Research and Health, vol. 34, no. 2, pp. 144-156, 2011. 
[16] J. Tsai, E. S. Ford, C. Li, and G. Zhao, "Past and current alcohol consumption patterns and elevations in serum hepatic enzymes among US adults," Addictive Behaviors, vol. 37, no. 1, pp. 78-84, 2012.

[17] C. D. C. Centers for Disease Control Prevention, National Health and Nutrition Examination Survey Data (NHANES), 2007-2008, Hyattsville, Md, USA, 2010.

[18] P. M. Krueger and E. M. Friedman, "Sleep duration in the united states: a cross-sectional population-based study," American Journal of Epidemiology, vol. 169, no. 9, pp. 1052-1063, 2009.

[19] M. A. Grandner, N. Jackson, J. R. Gerstner, and K. L. Knutson, "Dietary nutrients associated with short and long sleep duration. Data from a nationally representative sample," Appetite, vol. 64, pp. 71-80, 2013.

[20] N.I.A.A.A, "Rethinking drinking: what is at-risk or heavy drinking," http://rethinkingdrinking.niaaa.nih.gov/whatcountsdrink/whatsastandarddrink.asp.

[21] S. Liangpunsakul, "Relationship between alcohol intake and dietary pattern: findings from NHENES III," World Journal of Gastroenterology, vol. 16, no. 32, pp. 4055-4060, 2010.

[22] H. P. A. van Dongen, G. Maislin, J. M. Mullington, and D. F. Dinges, "The cumulative cost of additional wakefulness: doseresponse effects on neurobehavioral functions and sleep physiology from chronic sleep restriction and total sleep deprivation," Sleep, vol. 26, no. 2, pp. 117-126, 2003.

[23] M. A. Grandner and D. F. Kripke, "Self-reported sleep complaints with long and short sleep: a nationally representative sample," Psychosomatic Medicine, vol. 66, no. 2, pp. 239-241, 2004.

[24] B. Feige, H. Gann, R. Brueck et al., "Effects of alcohol on polysomnographically recorded sleep in healthy subjects," Alcoholism, vol. 30, no. 9, pp. 1527-1537, 2006.

[25] J. T. Arnedt, D. J. Rohsenow, A. B. Almeida et al., "Sleep following alcohol intoxication in healthy, young adults: effects of sex and family history of alcoholism," Alcoholism, vol. 35, no. 5, pp. 870-878, 2011.

[26] E. van Reen, T. L. Rupp, C. Acebo, R. Seifer, and M. A. Carskadon, "Biphasic effects of alcohol as a function of circadian phase," Sleep, vol. 36, no. 1, pp. 137-145, 2013. 


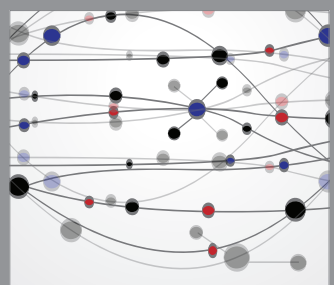

The Scientific World Journal
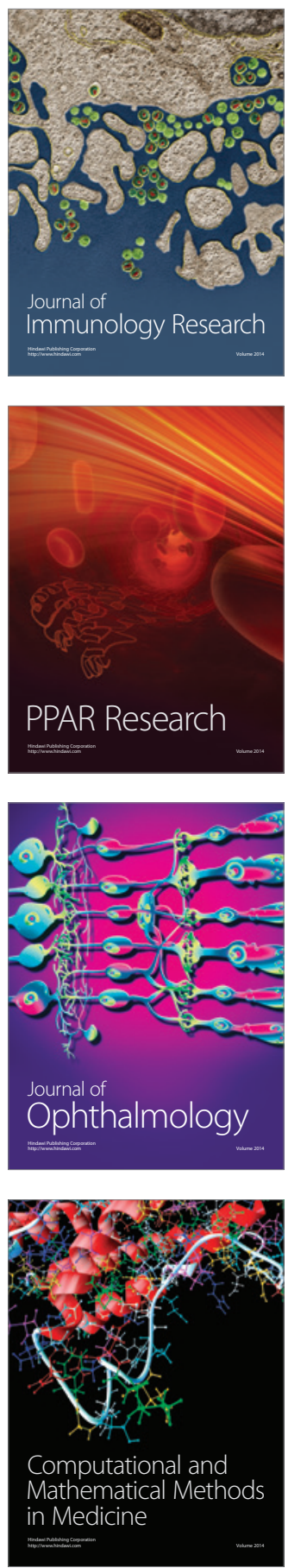

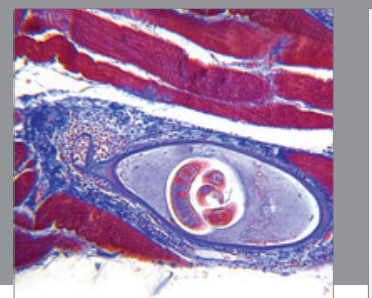

Gastroenterology

Research and Practice
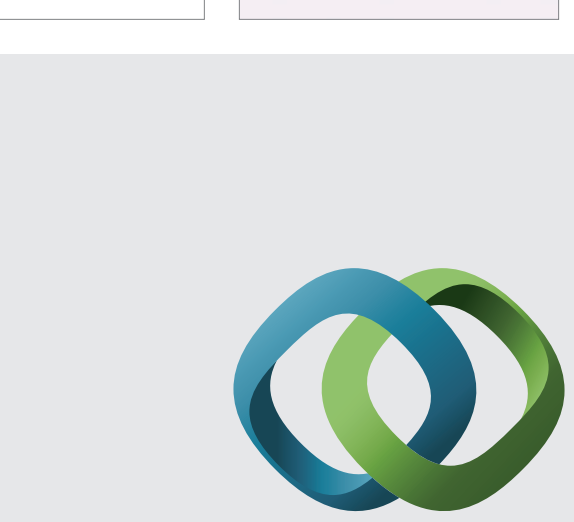

\section{Hindawi}

Submit your manuscripts at

http://www.hindawi.com
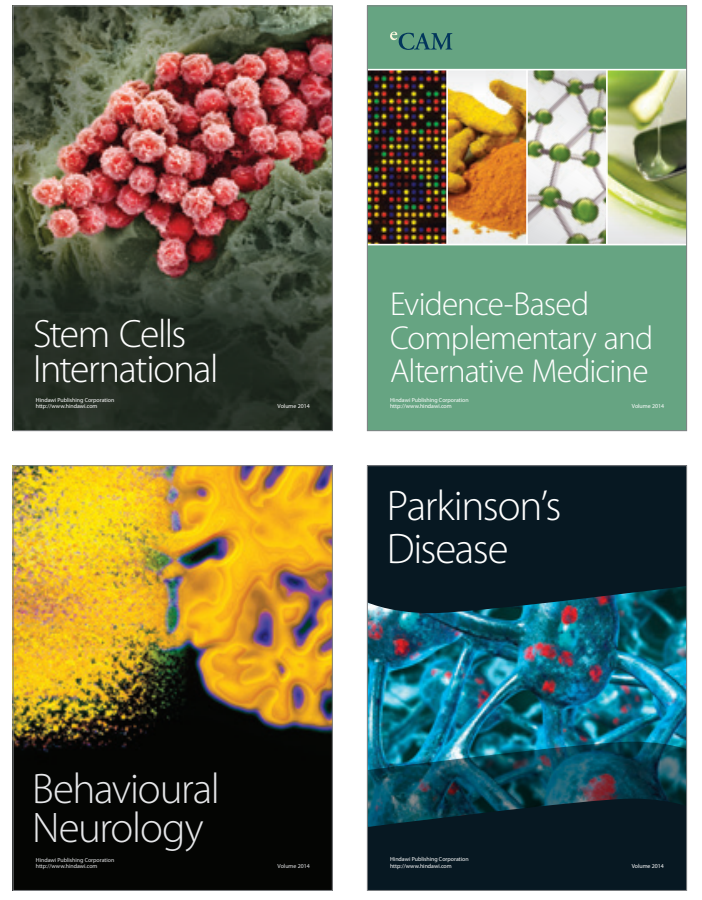
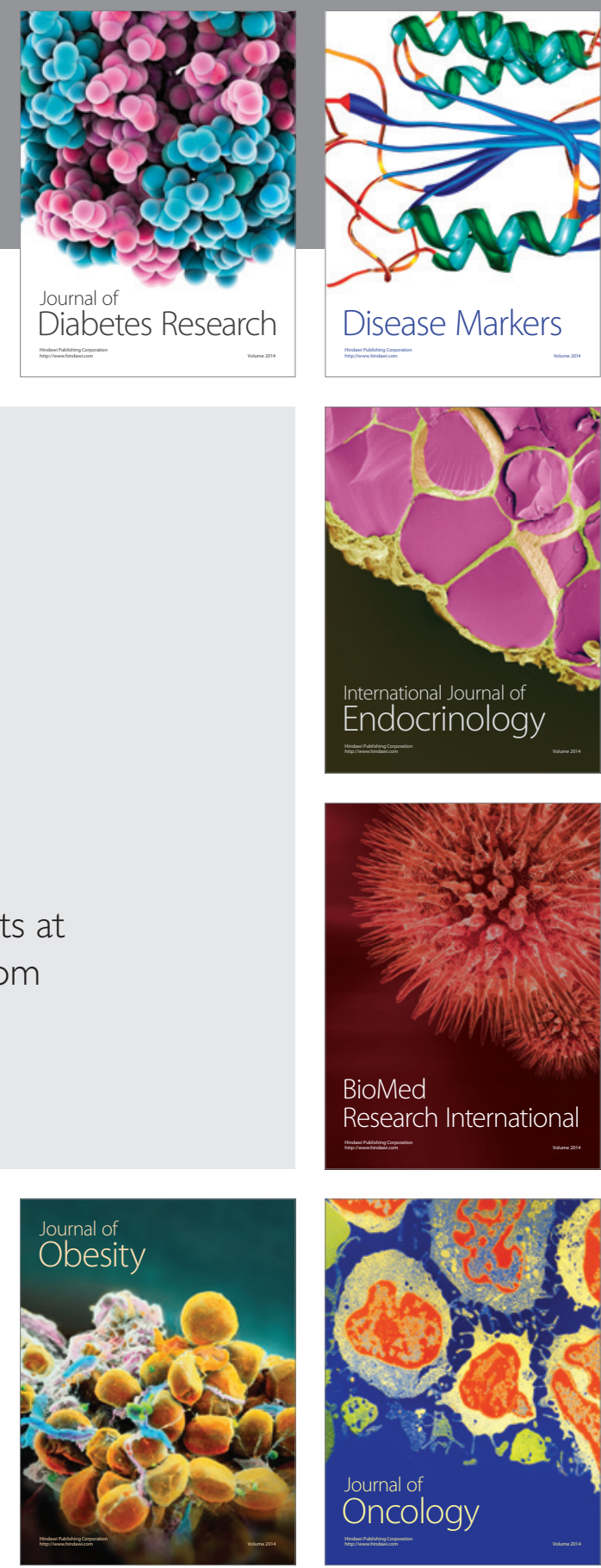

Disease Markers
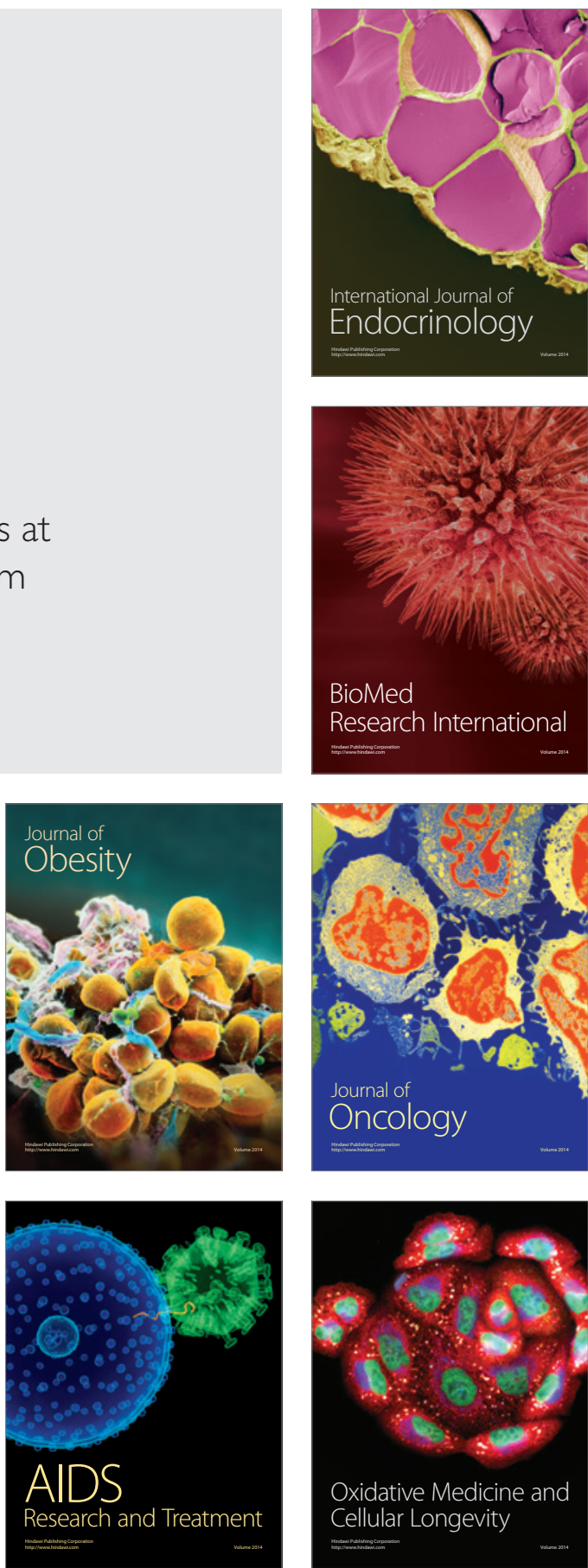